\title{
Duplicate Address Detection in Wireless Ad Hoc Networks Using Wireless Nature
}

\author{
Yu Chen and Eric Fleury \\ ARES/INRIA - INSA de Lyon, France \\ Eric.Fleury@inria.fr, ychen@cs.tamu.edu
}

\begin{abstract}
We consider duplicate address detection in wireless ad hoc networks under the assumption that addresses are unique in two hops neighborhood. Our approaches are based on the concepts of physical neighborhood views, that is, the information of physically connected nodes, and logical neighborhood views, which are built on neighborhood information propagated in networks. Since neighborhood information is identified by addresses, inconsistency of these two views might occur due to duplicate addresses. It is obvious that consistency of these two views on each node's neighborhood is necessary for a network to have unique addresses, while the sufficiency depends on the types of information contained in neighborhood views. We investigate different definitions of neighborhood views and show that the traditional neighborhood information, neighboring addresses, is not sufficient for duplication detection, while the wireless nature of ad hoc networks provides useful neighborhood information.
\end{abstract}

Keywords: duplicate address detection, wireless ad hoc networks, symmetry.

\section{Introduction}

A wireless ad hoc network is a set of wireless nodes which cooperatively and spontaneously form a network. Such a network provides a flexible means of communication without using any existing infrastructure or centralized administration. Significant research in ad hoc networks has focused on routing, the majority of which assume that nodes are configured a priori with a unique address. Since in ad hoc networks nodes join and leave at will, automated address assignment is required to dynamically configure nodes. In traditional networks, dynamic address assignment can be performed by a DHCP server [8]. But this solution is not suitable in ad hoc networks due to the unavailability of centralized servers. One alternative is to allow nodes to pick tentative addresses and the uniqueness of picked addresses is checked by some duplication detection mechanism; new tentative addresses are picked if duplications are detected [3, 17, 18, 19.

In this work, we focus on duplicate address detection in wireless ad hoc networks. Works on duplication detection have been proposed previously (e.g., 3, 17, 18, 19]). Many approaches assume the existence of global unique identification. Under this assumption, duplication can be detected by propagating 
associations of identifications and addresses. However, no global identification is truly unique; e.g., IEEE medium access control (MAC) addresses are not truly unique. One alternative is to create an identification randomly. The argument is that the probability of collision is small if the range of identifications is large enough. But propagating large-ranged identifications will cause large packet overhead. Thus relying on global uniqueness is not desirable. In our work, we consider detecting duplicate address based on local uniqueness: addresses are assume to be unique in two hops neighborhood. This assumption is made due to two facts. First, symmetry can prevent a problem to be solved in anonymous networks 2, 9, 10, thus some form of uniqueness is necessary; compared to the assumption of global unique identifications, our assumption is much weaker. Second, many algorithms have been proposed to assign addresses that are unique in two hops neighborhood (e.g., [1]).

We observe that protocols that are not aware of duplicate addresses behave as if all the packets from the same address are from the same node. For example, link state routing running on a node with address ip regards all the nodes that are connected to a node with address $i p$ as its neighbors. Thus if duplicate address exists, the view of link state routing on the neighborhood is different from the physical neighborhood view. Based on this observation, we propose the concepts of physical neighborhood views and logical neighborhood views. Informally, a physical neighborhood view of a node is information of nodes physically connected to it; examples include the number of neighbors, addresses of neighbors and distances to each neighbor. A logical neighborhood view is built based on neighborhood information identified by addresses: a node with address ip considers all the nodes that connect to a node that has address $i p$ as its neighbors and the view is built based on neighborhood information of all such "neighbors". For example, given a node that has address $i p$, the number of its neighbors in its physical view is the number of nodes physically connected to it, and in its logical view it is the number of nodes connected to a node that has address ip. More detailed example will be given later in Figure 1.

We consider duplicate address detection by comparing the physical and logical neighborhood views of each node. Logical neighborhood views can be built if each node propagates to all the others the state of each of its links, identified by ends' addresses. Since neighborhood information is required by most existing protocols and it usually contains two ends' addresses of each link, the overhead of our approaches depends on other information defined in neighborhood views. It is obvious that consistency of physical and logical views on each node's neighborhood is necessary for a network to have unique addresses, but whether it is sufficient depends on the types of information contained in neighborhood views. For example, if a neighborhood view is defined as the number of neighbors, it is sufficient only in a small class of networks.

We investigate different definitions of neighborhood views. We start from a traditional definition of neighborhood views, which consists of neighboring addresses. The idea of detecting duplication by comparing neighboring addresses has been proposed in PDAD-NH [19,18]. But no further investigation on the 
correctness is given. It is claimed "in case the sender of the link state packet is a common neighbor of the nodes with the same address, the conflict cannot be detected by PDAD-NH. Thus, conflicts in the two hops neighborhood must again be detected by other means". We take a close look at this approach under the assumption of unique address in two hops neighborhood and prove it fails in certain class of networks. We show this class of networks have the following properties: each existing address is assigned to the same number of nodes and there is a circle that has special properties. This class of networks might not be common in practice, but should not, therefore, be overlooked, since its existence indicates an important difference between wired and wireless networks. The properties of this class of networks provide strong hints for our second definition of neighborhood views, which also includes distance in $x$ and $y$ direction to each neighbor. We show that, under the assumption of unique addresses in two hops neighborhood, duplication can be detected if distance information satisfies certain accuracy, which means distance information can be represented in a small number of bits and overhead can be small. Note we do not assume the availability of strong position information such as GPS. Relative distance between neighboring nodes can be estimated by the signal strength or microwave or more sophisiticated techniques like microwave [14,20, 1, Neighbor or stronger distance information is used in many works on wireless networks [4, 14, 15.

\section{Related Work}

Dynamic Host Configuration Protocol (DHCP) [8] is commonly used for dynamic address assignment in traditional networks. Works on dynamic address assignment for ad hoc network include [12, 16, 13. Stateless approaches for local networks are proposed in [12] and [16], which require all nodes to be reachable in one-hop. In [13, addresses are treated as a shared resource and it is managed by a distributed mutual exclusion.

Solutions for duplication detection in ad hoc networks has been proposed previously (e.g. 3, 17, 18, 19]). In 3, each node has an fixed-length identifier which is randomly generated. A special message that includes nodes' address and identifier is diffused to the entire network; a node detects a duplicate address when it receives a message that has the same address as its own, but with a different identifier. Global unique or randomly generated keys are assumed in [17, in which duplication is detected by attaching key information in link state packets. The approach proposed in [17] successfully prevents packets from being delivered to wrong destinations. Most approaches for duplicate address detection require propagation of key information, which causes high packet overhead. Since lower protocol overhead is one of the most important design goals for wireless ad hoc networks, works have been done in achieving efficiency in terms of protocol overhead. Protocols proposed in [19] and 18] generate almost no protocol overhead: it detects address conflicts in a passive manner based on anomalies in routing protocol traffic. In particular, the idea of detecting duplication by comparing neighborhood information is proposed in approach PDAD-NH [19] [18]. 
However, no correctness proof is presented. In our work, we show this approach works in most networks, except a special class of networks; the existence of this class of networks indicates the different ability of wired and wireless networks in duplication detection using neighborhood information.

Much work has been done on anonymous networks in which no identifications are available $2,9,10,7,6$. Less work considers networks, especially wireless networks, with partial identifications. However, partial identification information, such as MAC addresses, are commonly available. Here we consider duplication detection using neighborhood information under the assumption of local uniqueness, which is not solvable in typical wired networks, but can be solved in ad hoc networks by using information provided by wireless nature.

\section{System Model and Overview}

We focus on stand-alone wireless ad hoc networks in which wireless nodes do not have access to a centralized server that could assign network-wide unique addresses. Instead of assuming global unique identifications, we consider duplication detection under the assumption that addresses are unique in two hops neighborhood. In our work, duplicate address is detected by each node comparing its physical neighborhood view and logical neighborhood view. In section 1, we have given an informal description of physical and logical neighborhood views. In the sequel, we focus on whether the consistency of physical neighborhood view and logical neighborhood view on every node is sufficient for a network to have unique addresses. If it is sufficient, in a network that has duplicate address, at least one node will detect duplication and it can inform other nodes. In our work, we examine two definitions; each definition has its own assumptions on neighborhood knowledge.

Physical neighborhood views are built based on neighborhood knowledge that are assumed to be available, thus no packet overhead is caused. But building logical neighborhood views requires propagation of neighborhood information, which causes packet overhead. We assume each node that has address ip generates packets $\left\langle i p, i p^{\prime}\right.$, link_state $\rangle$ for each neighbor that has address $i p^{\prime}$; the field link_state will be specified by the specific approach. We borrow the name from link state routing and call these packets as link state packets. Since neighborhood information is required by most protocols and how to propagate this information is out of the scope of this paper, we assume each node receives link state packets from all the other nodes without going into details of how these packets are propagated. Since most neighborhood information contains two ends' addresses of each link, we evaluate the overhead of each approach based on the packet complexity of field link_state in link state packets.

In the first approach (section 4), we assume neighboring addresses are available and neighborhood view is defined as a set of neighboring addresses. No overhead is introduced. We prove this information is not sufficient and this approach fails in certain class of networks; in this class of networks, all the existing addresses are assigned to the same number of nodes and there is a circle in 
which the sequence of nodes' addresses consists of repeated patterns. Based on this property, we propose our second definition (section (5). We observe that, due to its wireless nature, neighbor distance information is available in ad hoc networks. In our second approach, neighborhood view is defined as distances in $x$ and $y$ direction to each neighbor, together with ends' addresses of each link. Overhead of this approach is distant information in link state packets. We show that duplication can be detected if nodes that have the same address are not too "close"; the meaning of "being close" depends on the accuracy of neighbor distance. The allowance of inaccuracy implies a small number of bits can be used to represent distance information. Based on the assumption that addresses are unique in two hops neighborhood, only small overhead is required.

Before we present our definitions of neighborhood views, we introduce the concept of addresses map, which is used in our analysis. Informally, addresses map is a view of a network in which all the nodes with the same address are combined into one. An example is given in Figure 1.

Definition 1. (Addresses Map) Given a network G, its addresses map is a graph such that each vertex is a distinct existing address and there is an edge between addresses $i p_{1}$ and $i p_{2}$ iff there exits link state packet $\left\langle i p_{1}, i p_{2}\right\rangle$ or $\left\langle i p_{2}, i p_{1}\right\rangle$.

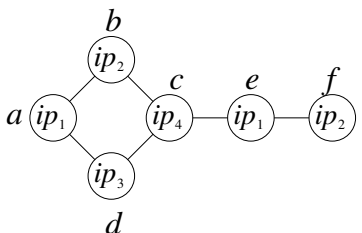

Network

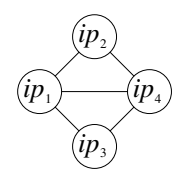

Map of Addresses

\begin{tabular}{|c|l|}
\hline node & links identified by ends' addresses \\
\hline$a$ & $\left\langle i p_{1}, i p_{2}\right\rangle,\left\langle i p_{1}, i p_{3}\right\rangle$ \\
\hline$b$ & $\left\langle i p_{2}, i p_{1}\right\rangle,\left\langle i p_{2}, i p_{4}\right\rangle$ \\
\hline$c$ & $\left\langle i p_{4}, i p_{1}\right\rangle,\left\langle i p_{4}, i p_{2}\right\rangle,\left\langle i p_{4}, i p_{3}\right\rangle$ \\
\hline$d$ & $\left\langle i p_{3}, i p_{1}\right\rangle,\left\langle i p_{1}, i p_{4}\right\rangle$ \\
\hline$e$ & $\left\langle i p_{1}, i p_{4}\right\rangle,\left\langle i p_{1}, i p_{2}\right\rangle$ \\
\hline$f$ & $\left\langle i p_{2}, i p_{1}\right\rangle$ \\
\hline
\end{tabular}

\begin{tabular}{|c|l|}
\hline address $i p$ & neighbors of $i p$ in the map \\
\hline$i p_{1}$ & $i p_{2}, i p_{3}, i p_{4}$ \\
\hline$i p_{2}$ & $i p_{1}, i p_{4}$ \\
\hline$i p_{3}$ & $i p_{1}, i p_{4}$ \\
\hline$i p_{4}$ & $i p_{1}, i p_{2}, i p_{3}$ \\
\hline
\end{tabular}

Fig. 1. An Example of Addresses Map

We use terms "addresses" and "edges" to refer to vertices and links in the addresses map respectively, and "nodes" and "links" to refer to vertices and links in a network respectively. We say a link connects two addresses $i p$ and $i p^{\prime}$ if its two ends have addresses $i p$ and $i p^{\prime}$. The lemma below shows a necessary condition for a network to have duplicate address. Proof is not provided due to limited space; it can be found in full paper [5]. Note the existence of circles in its addresses map is not a sufficient condition for duplication to exist in a network.

Lemma 1. Given a network in which addresses are unique in two hops neighborhood, if no circle exists in its addresses map, then no duplicate address exists. 


\section{Duplication Detection Using Neighboring Addresses}

In this section, the only assumption on neighborhood knowledge is that each node knows its neighboring addresses. The view of a node's neighborhood is defined as the set of neighboring addresses. Since no information except ends' addresses of each link is required to build logical neighborhood views, link state packets have form of $\left\langle i p, i p^{\prime}\right\rangle$ and no overhead is caused.

Definition 2. Given a network and a node $n$ that has address ip, we define

- physical neighborhood view of $n \equiv$ the set of addresses of nodes that are physically connected to $n$.

- logical neighborhood view of $n \equiv\left\{i p^{\prime} \mid \exists\right.$ link state packet $\left.\left\langle i p, i p^{\prime}\right\rangle\right\}$

The term "view" is used in this section according to this definition. Table 1 describes physical and logical neighborhood views of the network in Figure 1, in which this approach works. However, special symmetry can prevent this approach from detecting duplications. Counterexamples are given in Figure 2, all the nodes in Network 1 and Network 2 have consistent views, but duplications exist in both networks. Note these two networks have the same addresses map.

Table 1. An Example: Views of Neighborhood of Network in Figure 1

\begin{tabular}{|c|l|l|c||c|l|l|c|}
\hline node & physical view & logical view & Consist. & node & physical view & logical view & Consist. \\
\hline$a$ & $i p_{2}, i p_{3}$ & $i p_{2}, i p_{3}, i p_{4}$ & False & $b$ & $i p_{1}, i p_{4}$ & $i p_{1}, i p_{4}$ & True \\
\hline$c$ & $i p_{1}, i p_{2}, i p_{3}$ & $i p_{1}, i p_{2}, i p_{3}$ & True & $d$ & $i p_{1}, i p_{4}$ & $i p_{1}, i p_{4}$ & True \\
\hline$e$ & $i p_{2}, i p_{4}$ & $i p_{2}, i p_{3}, i p_{4}$ & False & $f$ & $i p_{1}$ & $i p_{1}, i p_{4}$ & False \\
\hline
\end{tabular}

Now we investigate the properties of networks in which this approach fails. The lemma below shows that in such a network, addresses are distinct in the shortest path connecting any two different addresses. Due to limited space, proof is not provided and it can be found in [5].

Lemma 2. Consider a network in which addresses are unique in two hops neighborhood and views are consistent on every node. Given any two addresses ip $_{x}$ and $i p_{y}, i p_{x} \neq i p_{y}$, nodes in a shortest path that connects $i p_{x}$ and $i p_{y}$ have distinct addresses.

The lemma below states that in such networks, given a path in which nodes have distinct addresses, there are $t$ distinct paths that have same sequence of addresses, where $t$ depends on the number of nodes that have the same address.

Lemma 3. Consider a network in which addresses are unique in two hops neighborhood and views are consistent on every node. Given a path path in $_{\text {in }}$ which all the nodes have distinct addresses. Let $t$ be the number of nodes that have address

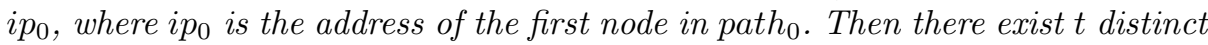
paths that have the same sequence of addresses as path . 


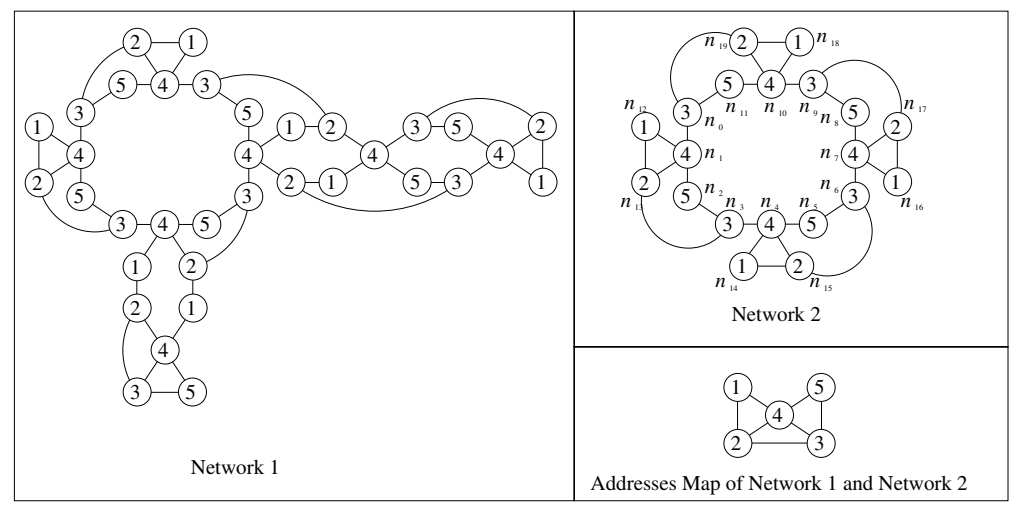

Fig. 2. Examples in which views are consistent \& duplicate addresses exist

Proof. Let path $_{0}$ be $\left\langle n_{0}^{0}, n_{0}^{1}, \ldots, n_{0}^{k}\right\rangle$. We denote the address of $n_{0}^{i}$ by $i p_{i}$. We construct $t$ paths by induction. For some $s \in[0, t-2]$, we assume there are

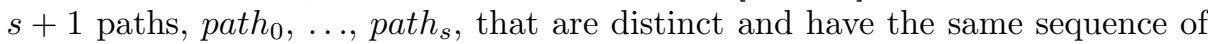
addresses as path $h_{0}$. Note it is true when $s=0$. We construct path $h_{s+1}$ as follows.

We denote nodes in path $_{0}, \ldots$, path $_{s}$ by path $s_{[0, s]}$. Since there are $t$ nodes that have address $i p_{0}$ and only $s+1 \leq t-1$ of them are in $p a t h s_{[0, s]}$, there is at least one node that has address $i p_{0}$ and is not in $p a t h s_{[0, s]}$. Let this node be $n_{s+1}^{0}$.

Now we construct the rest of this path by induction. For $i \in[0, k-1]$, assume there is a path $\left\langle n_{s+1}^{0}, \ldots, n_{s+1}^{i}\right\rangle$ such that: (1) $\forall l \in[0, i]$, the address of $n_{s+1}^{l}$ is $i p_{l}$; and (2) all the selected nodes, that is, $\left\{n_{s+1}^{0}, \ldots, n_{s+1}^{i}\right\} \bigcup \operatorname{path}_{[0, s]}$, are distinct. We select $n_{s+1}^{i+1}$ as follows (Figure 3). Since views are consistent on every node and $n_{s+1}^{i}$ and $n_{0}^{i}$ have the same address, $n_{s+1}^{i}$ and $n_{0}^{i}$ have the same set of neighboring addresses. Since $n_{0}^{i}$ has a neighbor $n_{0}^{i+1}$ that has address $i p_{i+1}$, $n_{s+1}^{i}$ also has a neighbor that has address $i p_{i+1}$, denoted by $n^{\prime}$. Now we show $n^{\prime}$ is not among the selected nodes. Among all the selected nodes, only nodes in $\left\{n_{0}^{i+1}, \ldots, n_{s}^{i+1}\right\}$ have address $i p_{i+1}$. If $n^{\prime}$ is one of them, then $n^{\prime}$ is connected to $n_{l}^{i}$ for some $l \in[0, s]$. So $n^{\prime}$ is connected to $n_{l}^{i}$ and $n_{s+1}^{i}$, which have the same address $i p_{i}$. It contradicts to the assumption of unique addresses in two hops neighborhood. Since $n^{\prime}$ has not been selected and it has address $i p_{i+1}$, it can be selected as $n_{s+1}^{i+1}$ and the lemma is proved.

Based on these two lemmas, the following theorem states that all the existing addresses are assigned to the same number of nodes. An interesting implication is that a network with a prime number of nodes does not have duplicate address if views are consistent on every node. We define the duplicate degree of such a network as the number of nodes that take the same existing address.

Theorem 1. Consider a network in which addresses are unique in two hops neighborhood and views are consistent on every node. Given an address ip, denoting the number of nodes that take ip as its address by $f(i p)$, we have $f\left(i p^{\prime}\right)=f\left(i p^{\prime \prime}\right)$ for any two addresses $i p^{\prime}$ and $i p^{\prime \prime}$ that exist in the network. 


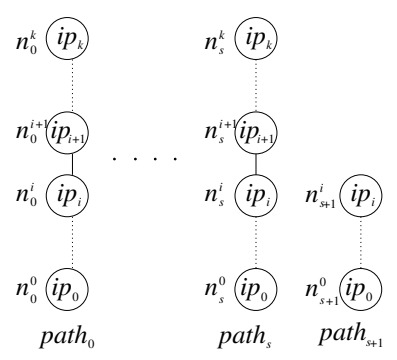

Fig. 3. Proof of Lemma 3

Proof. Assume in contradiction that there exist addresses $i p_{x}$ and $i p_{y}$ such that the number of nodes that have address $i p_{x}$ is $s$ and the number of nodes that have address $i p_{y}$ is $t$, where $s, t \geq 1$ and $s>t$. Consider all the pairs $x^{\prime}$ and $y^{\prime}$ such that $x^{\prime}$ has address $i p_{x}$ and $y^{\prime}$ has address $i p_{y}$. Let $x$ and $y$ be the closest

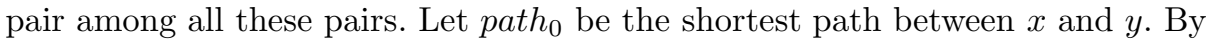
Lemma 2, the addresses of nodes in path $_{0}$ are distinct. By lemma 3, there are $s$ paths with the same sequence of addresses as path $h_{0}$ and all the nodes in these paths are distinct. So there are $s$ nodes that have the same address as $y$, which contradicts to that only $t, t<s$, nodes has address $i p_{y}$.

The above theorem examines the connection between the number of nodes and that of addresses. Now we take a close look at the connection between the topology of a network and that of its addresses map. In particular, given a subgraph $S_{A}$ of its addresses map, we examine the subgraph of a network that is "relevant" to $S_{A}$. Informally, a node is relevant if it has an address in $S_{A}$ and a link is relevant if an edge connecting its two ends' addresses exists in $S_{A}$. We say such a subgraph is expanded by $S_{A}$. The formal definition is given below.

Definition 3. (Expanded Subgraph): Given a network $G$ and a subgraph $S_{A}$ of its addresses map, we consider a subgraph $S_{G}$ of $G$ that satisfies: nodes in $S_{G}$ are the nodes that have addresses in $S_{A}$, and there is an link between nodes $x$ and $y$ in $S_{G}$ iff there is an edge between the address of $x$ and the address of $y$ in $S_{A}$. We say $S_{G}$ is the subgraph that is expanded by $S_{A}$.

In Theorem [2, we examine addresses that are organized in a circle in the addresses map. We show that the subnetwork expanded by it consists of a set of circles. Furthermore, if duplication exists, there is a "minimal" circle in the addresses map which expands a subgraph that contains a circle with duplicate addresses; the existence of such a circle provides strong hints for our second approach. A "minimal circle" is defined below. For example, in Network 2 of Figure 2 circle $\langle 3,4,5,3\rangle$ is minimal while circle $\langle 1,2,3,4,1\rangle$ is non-minimal.

Definition 4. (Minimal Circle): Given a graph $G$, a circle cir is minimal iff there exists a node $x$ in cir such that cir is the shortest circle that contains $x$. 


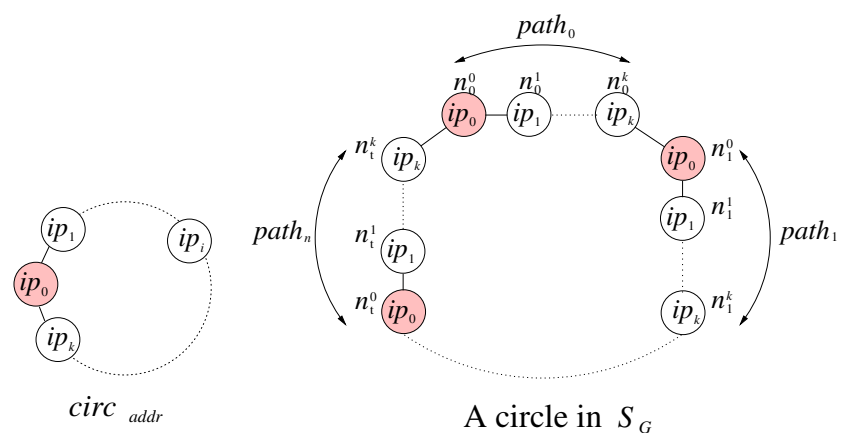

Fig. 4. Theorem 2

Theorem 2. Consider a network $G$ in which addresses are unique in two hops neighborhood and views are consistent on every node. Given any circle circ $_{a d d r}=\left\langle i p_{0}, i p_{1}, \ldots, i p_{k}, i p_{0}\right\rangle$ in the addresses map, the subgraph $S_{G}$ of $G$ that is expanded by circ $_{a d d r}$ consists of a set of circles, and each circle has the form of

$$
\text { path }_{0} \circ \text { path }_{1} \cdots \circ \text { path }_{s-1} \circ\left\langle n_{0}\right\rangle
$$

where path $_{i}$ is a path that has sequence of addresses $\left\langle i p_{0}, \ldots, i p_{k}\right\rangle, n_{0}$ is the first node in path p $_{0}$ and $s \geq 1$ (Figure 4).

Furthermore, if duplicate address exists, there exists a minimal circle in the addresses map whose expanded subgraph in $G$ contains a circle that has $s>1$ in the above form.

Proof is not provided due to limited space; it can be found in [5]. We consider Network 2 in Figure 2 as an example. The duplicate degree is 4 . The subgraph expanded by the circle of addresses $\langle 1,2,4,1\rangle$ consists of four circles. The subgraph expanded by a minimal circle $\langle 3,4,5,3\rangle$ is one circle with $s=4:\left\langle n_{0}, n_{1}, n_{2}\right.$, $\left.n_{3}, n_{4}, n_{5}, n_{6}, n_{7}, n_{8}, n_{9}, n_{10}, n_{11}\right\rangle$. A non-minimal circle $\langle 1,2,3,4,1\rangle$ also expands a subgraph that has $s=4:\left\langle n_{12}, n_{13}, n_{3}, n_{4}, n_{14}, n_{15}, n_{6}, n_{7}, n_{16}, n_{17}, n_{9}, n_{10}, n_{18}\right.$, $\left.n_{19}, n_{0}, n_{1}, n_{12}\right\rangle$.

\section{Duplication Detection Using Neighbor Distance}

In Theorem 2, we show that if duplication exists and views defined as neighboring addresses are consistent at every node, there exists in the network a circle which consists of pattens of nodes that have the same sequence of addresses. For example, a pattern in Figure 4 is $\left\langle n_{i}^{0}, \ldots, n_{i}^{k}, n_{i+1}^{0}\right\rangle$ (here we write patterns in such a way that the first node of the next pattern is the last node of the last pattern). In order to form a circle, either the distance between two ends of each pattern is zero, which means two nodes with address $i p_{0}$ are at the same location; or patterns do not have the same shapes and orientations, since otherwise the end of the last pattern cannot go back to the beginning of the first 
pattern. Since the sequence of addresses is the same for all the patterns, difference in shapes and orientations means relative distances between neighbors differ at nodes with the same address. Thus if neighbor distance information is included, inconsistency of neighborhood views will be detected. Since in practice accurate distance information might not be available due to inaccuracy in measurement or limitation in the number of bits to represent distance information, we consider duplication detection using inaccurate distance information with bounded error, instead of accurate information. Note in this approach, the only modification of the original link state routing is to attach relative distances to neighbors in link state packets.

We denote the real $x$-coordinate ( $y$-coordinate resp.) of node $n$ by $x_{\text {coor }}(n)$ $\left(y_{\text {coor }}(n)\right.$ resp. $)$, and the real distance from node $n$ to node $n^{\prime}$ in $x$-direction (y-direction resp.) by $\operatorname{dis}_{X}\left(n, n^{\prime}\right)$ ( $\operatorname{dis}_{Y}\left(n, n^{\prime}\right)$ resp.). We assume each node $n$ has distance information to each neighbor $n^{\prime}$ in $x$-direction and $y$-direction, denoted by $d i s_{X \_i n f}\left(n, n^{\prime}\right)$ and $d i s_{Y \_i n f}\left(n, n^{\prime}\right)$ respectively. Node $n$ that has address ip generates link state packet for each of its neighbor $n^{\prime}$ that has address $i p^{\prime}$ in the form of $\left\langle i p, i p^{\prime}, d_{x}, d_{y}\right\rangle$, where $d_{x}=\operatorname{dis}_{X_{-} i n f}\left(n, n^{\prime}\right)$ and $d_{y}=d i s_{Y \_i n f}\left(n, n^{\prime}\right)$. Note distance information obtained by each node might differ from the real information. Let $e_{r r}$ be the bound on distance errors defined as follows: $\forall n, \forall$ neighbor $n^{\prime}$ of $n,\left|d i s_{X \_i n f}\left(n, n^{\prime}\right)-\operatorname{dis}_{X}\left(n, n^{\prime}\right)\right| \leq e_{r r}$ and $\left|d i s_{Y \_i n f}\left(n, n^{\prime}\right)-\operatorname{dis}_{Y}\left(n, n^{\prime}\right)\right| \leq e_{r r}$. Physical and logical neighborhood views are defined below; the term "view" is used in this section according to this definition.

Definition 5. Given a network and a node $n$ that has address ip, we define

- physical neighborhood view of $n \equiv\left\{\left\langle i p^{\prime}, \operatorname{dis}_{X_{\_} \text {inf }}\left(n, n^{\prime}\right), \operatorname{dis}_{Y_{\text {_inf }}}\left(n, n^{\prime}\right)\right\rangle \mid i p^{\prime}\right.$ is the address of a node $n^{\prime}$ that is physically connected to $\left.n\right\}$

- logical neighborhood view of $n \equiv\left\{\left\langle i p^{\prime}, d_{x}, d_{y}\right\rangle \mid \exists\right.$ link state packet $\left\langle i p, i p^{\prime}\right.$, $\left.\left.d_{x}, d_{y}\right\rangle\right\}$

The next theorem states the impact of distance errors on duplication detection. In a network with duplicate addresses, if there exists inconsistency in neighboring addresses, inconsistent views will surely be detected; otherwise by the theorem below, duplication will be detected if any two nodes with the same addresses are not too close.

Theorem 3. Consider a network in which addresses are unique in two hops neighborhood and nodes that have the same address have the same set of neighboring addresses. At least one node has inconsistent views if any two nodes that have the same address are away at least $2 k \cdot e_{r r}$ in both $x$-direction and $y$-direction, where $e_{r r}$ is an upper bound on errors in distance information and $k$ is the length of a special circle defined in the second part of Theorem 2 .

Proof. By Theorem 2] there is a cycle, $\left\langle i p_{0}, \ldots, i p_{k-1}, i p_{0}\right\rangle$, in the address map such that there exists a circle in the network $\left\langle n_{0}^{0}, n_{0}^{1}, \ldots, n_{0}^{k-1}, n_{1}^{0}, n_{1}^{1}, \ldots\right.$, $\left.n_{1}^{k-1}, \ldots, n_{s-1}^{0}, n_{s-1}^{1}, \ldots, n_{s-1}^{k-1}, n_{0}^{0}\right\rangle$, where $s \geq 1$ and the address of $n_{j}^{i}$ is $i p_{i}$ 


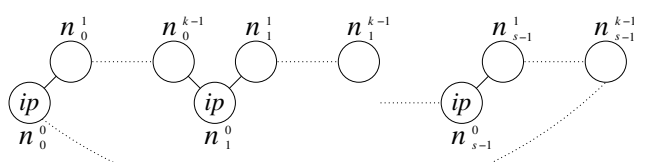

Fig. 5. Proof of Theorem 3

$\forall j \in[0, s-1]$ (Figure 5). We assume in contradiction that all the nodes have consistent views. We define two denotations: (1) the real distance in $x$-direction from $n_{i}^{0}$ to $n_{(i+1) \% s}^{0}: \operatorname{seg}_{X i}=\sum_{j=0}^{k-2} \operatorname{dis}_{X}\left(n_{i}^{j}, n_{i}^{j+1}\right)+\operatorname{dis} s_{X}\left(n_{i}^{k-1}, n_{(i+1) \%_{s}}^{0}\right)$; and (2) $\operatorname{seg}_{X \_i n f}=\sum_{j=0}^{k-2} d i s_{X \_i n f}\left(n_{i}^{j}, n_{i}^{j+1}\right)+\operatorname{dis}_{X \_i n f}\left(n_{i}^{k-1}, n_{(i+1) \% s}^{0}\right)$. Note the value of $\operatorname{seg}_{X \_i n f}$ does not depend on $i$, because for all $i$, dis $s_{X \_i n f}\left(n_{i}^{j}, n_{i}^{j+1}\right)$ has the same value since $n_{i}^{j}=i p_{j}$ and $n_{i}^{j+1}=i p_{j+1}$ and views are consistent on all the nodes.

By the definition of $e_{r r}$, we have $\left|\operatorname{seg}_{X i}-\operatorname{seg}_{X \_i n f}\right| \leq k e_{r r}$. Since $\sum_{i=0}^{s-1} \operatorname{seg}_{X i}=0$, we have $\operatorname{seg}_{X_{-i n f}} \in\left[-k e_{d}, k e_{d}\right]$, that is, $\operatorname{seq}_{X i} \in\left[-2 k e_{d}, 2 k e_{d}\right]$. So nodes $n_{i}^{0}$ and $n_{(i+1) \% s}^{0}$ are within $2 k e_{r r}$ in $x$-direction. Similarly, we can prove $n_{i}^{0}$ and $n_{(i+1) \% s}^{0}$ are within $2 k e_{r r}$ in $y$-direction. So there are two nodes that have the same address and are within distance $2 k e_{r r}$ in both $x$ and $y$ direction. Contradiction!

Now we discuss how nodes decide the number of bits to represent distance information if accurate distance information is available. We consider a network in which transmission range of nodes is $R$. Letting $d_{x}$ ( $d_{y}$ resp.) be the distance within any two nodes that have the same address in $x$-direction $(y$-direction resp.), we have $\max \left\{\left|d_{x}\right|,\left|d_{y}\right|\right\} \geq \frac{R}{\sqrt{2}}$ by the assumption that addresses are unique within in two hops neighborhood. In order to detect duplication, we require $2 k e_{r r} \leq \frac{R}{\sqrt{2}}$, that is, $e_{r r} \leq \frac{R}{2 \sqrt{2} k}$. If $b$ bits are used to represent distance information in link state packets, we have $e_{r r} \leq \frac{R}{2^{b}}$. So all duplications can be detected if $\frac{R}{2^{b}} \leq \frac{R}{2 \sqrt{2} k}$, that is, $b \geq 1.5 \log k$. Nodes can get an upper bound on $k$ by checking lengths of minimal circles in the addresses map; the the "minimal" property of such a circle shown in Theorem 2 implies high possibility of a small $k$. Note a trivial upper bound on $k$ is the number of addresses, which is smaller than the number of nodes or the length of some assumed global unique identification.

\section{Conclusion}

We investigated duplicate address detection under the assumption that addresses are unique within two hops neighborhood. We propose two definitions of neighborhood views and duplication detection is done by comparing the physical and logical neighborhood views of each node. We show traditional neighborhood information, neighboring addresses, is not sufficient to detect duplicate address, while duplication can be detected by using neighbor distance information that satisfies certain accuracy. 


\section{References}

1. A.Benlarbi, J. Cousin, R. Ringot, A. Mamouni, and Y. Leroy. Interferometric positioning systems by microwaves. In Proc. Microwaves Symp., Tetuan, Morocco, 2000.

2. P. Boldi and S. Vigna. Universal dynamic synchronous self-stabilization. Distributed Computing, 15-3:137-153, 2002.

3. S. Boudjit, A. Laouiti, P. Muhlethaler, and C. Adjih. Duplicate address detection and autoconfiguration in olsr. In $S N P D-S A W N$ '05, pages 403-410, Washington, DC, USA, 2005. IEEE Computer Society.

4. J. Cartigny, D. Simplot, and I. Stojmenovic. Localized minimum-energy broadcasting in ad-hoc networks. In INFOCOM 2003, 2003.

5. Y. Chen and E. Fleury. Duplicate Address Detection in Wireless Ad Hoc Networks Using Wireless Nature, Research Report, to appear, INRIA, Feb. 2006.

6. B. S. Chlebus, L. Gasieniec, A. Ostlin, and J. M. Robson. Deterministic radio broadcasting. In Automata, Languages and Programming, pages 717-728, 2000.

7. A. E. F. Clementi, A. Monti, and R. Silvestri. Selective families, superimposed codes, and broadcasting on unknown radio networks. In Proc. 12th Annual ACMSIAM Symposium on Discrete Algorithms, pages 709-718, Washington, DC, 2001.

8. R. Droms. Dynamic host configuration protocol, 1997.

9. T. K. M. Yamashita. Computing on anonymous networks: Part i - characterizing the solvable cases. IEEE Transactions on Parallel and Distributed Systems, 7(1):69-89, 1998.

10. T. K. M. Yamashita. Computing on anonymous networks: Part i - decision and membership problems. IEEE Transactions on Parallel and Distributed Systems, $7(1): 90-96,1998$.

11. N. Mitton, E. Fleury, I. Gurin-Lassous, B. Sricola, and S. Tixeuil. On fast randomized colorings in sensor networks. technical report LRI-1416, INRIA, Jun. 2005 .

12. M. Mohsin and R. Prakash. An ip address configuration algorithm for zeroconf mobile multihop ad hoc networks. In Int'l. Wksp. Broadband Wireless Ad Hoc Networs and Services, Sept 2002.

13. S. Nesargi and R.Prakash. Manetconf: Configuration of hosts in a mobile ad hoc network. In INFOCOM 2002, June 2002.

14. S. Ni, Y. Tseng, Y. Chen, and J. Sheu. The broadcast storm problem in a mobile ad hoc network. In Proc. 5th Annual ACM/IEEE International Conference on Mobile Computing and Networking, pages 151-162, Seattle, WA, 1999.

15. R. C. Shah and J. M. Rabaey. Energy aware routing for low energy ad hoc sensor networks. In IEEE WCNC, 2002.

16. S. Thomoson and T. Narten. Ipv6 stateless address autoconfiguration. RFC 2462, Dec 1998.

17. N. H. Vaidya. Weak duplicate address detection in mobile ad hoc networks. In Proc. 3rd ACM International Symposium on Mobile Ad Hoc Networking and Computing, pages 206-216. ACM Press, 2002.

18. K. Weniger. Passive duplicate address detection in mobile ad hoc networks. In IEEE WCNC, New Orleans, LA, Mar 2003.

19. K. Weniger. Pacman: Passive autoconfiguration for mobile ad hoc networks. IEEE Journal on Selected Areas in Communications, 23(3):507-519, 2005.

20. E. Wesel. Wireless Multimedia Communications: Networking Video, Voice, and Data. Addison-Wesley, Reading, MA, 1998. 